\title{
MULTIPLE CANONICAL DECOMPOSITIONS OF FAMILIES OF OPERATORS AND A MODEL OF QUASINORMAL FAMILIES
}

\author{
XIMENA CATEPILLÁN, MAREK PTAK, AND WACLAW SZYMAŃSKI
}

(Communicated by Palle E. T. Jorgensen)

Dedicted to the memory of our friend Domingo Herrero

\begin{abstract}
A general method of canonical decompositions of several operatorvalued functions (operators) is presented. A model of a family of doubly commuting quasinormal operators is constructed.
\end{abstract}

\section{INTRODUCTION}

The idea of decomposing an operator (a family of operators, an operatorvalued function) into parts, which are easier to investigate than the original operator, is fundamental to the theory of operators.

The so-called canonical decomposition is one of many known kinds of decompositions. Wold [11], studying stationary stochastic processes, discovered the decomposition of an isometry into the unitary and the completely nonunitary parts, which has since been referred to as the Wold decomposition of an isometry and is the first example of a canonical decomposition. A general theory of canonical decompositions of a single operator (more generally, an operatorvalued function) with respect to a single property has been developed in [7]. Following Wold's idea, Stocinski [6] studied canonical decompositions of two commuting isometries. Using in an essential manner the isometric-unitary properties, he established a criterion for "the best" analogue of the Wold decomposition to exist and found an ingenious example of two commuting isometries without such decomposition [6, Example 1].

In $\S 2$ we present a general method of constructing canonical decompositions of several operator-valued functions (operators, in particular) with respect to several properties, which, in order to make such decompositions possible, not only should behave well themselves (as in [7]) but also should behave well with respect to each other (Theorem 2.2). This multiple canonical decomposition method is based on results of [7].

Canonical decompositions are often the first step in constructing models of operators. Of particular interest here is the model of a single quasinormal

Received by the editors November 9, 1992; part of this paper was presented to the 99th Annual Meeting of the AMS, San Antonio, Texas, 1993.

1991 Mathematics Subject Classification. Primary 47A65, 47B20.

The first author was partially supported by a grant from Millersville University. 
operator discovered by Brown in [2]. This model inspired $\S 3$, in which, using a multiple canonical decomposition of a family of doubly commuting operators (Corollary 2.8) together with Słocinski's complete analysis of doubly commuting shifts [6], we establish a model of a family of doubly commuting quasinormal operators.

$B(H)$ stands for the algebra of all linear, bounded operators in a Hilbert space $H . I$ denotes the identity operator. A projection is an operator $E \in$ $B(H)$ that satisfies $E=E^{2}=E^{*}$. If $\mathscr{S} \subset B(H)$, then $\mathscr{S}^{\prime}$ denotes the commutant of $\mathscr{S}$ and $W^{*}(\mathscr{S})$ denotes the von Neumann algebra generated by $\mathscr{S}$. Two projections $E, F$ in a von Neumann algebra $\Re \subset B(H)$ are equivalent with respect to $\mathfrak{R}$ if there is a partial isometry $V \in \Re$ such that $V^{*} V=E, V V^{*}=F$. A family $\left(T_{j}\right)_{j \in J} \subset B(H)$ is unitarily equivalent to a family $\left(S_{j}\right)_{j \in J} \subset B(K)$, where $K$ is a Hilbert space, if there is a unitary mapping $\phi: K \rightarrow H$ such that $\phi S_{j}=T_{j} \phi, j \in J . \mathbb{N}$ stands for the set of all nonnegative integers.

\section{Multiple CANONiCAl DECOMPOSITIONS}

First the terminology and necessary results will be recalled from [7].

Let $\Omega$ be a set, let $\varphi: \Omega \rightarrow B(H)$ be a function, and let $(W)$ be a property concerning $\varphi$. A projection $E \in B(H)$ is called $(W)$-reducing for $\varphi$ if $E \in \varphi(\Omega)^{\prime}$ and the function $u \rightarrow \varphi(u)_{\mid E H}$ has the property $(W)$. Denote by $\mathscr{P}$ the family of all $(W)$-reducing projections for $\varphi$. A property $(W)$ is called hereditary if, for each $E \in \mathscr{P}$, for each projection $F \in \varphi(\Omega)^{\prime}, F \leq E$ implies $F \in \mathscr{P}$. Let $Q \in \varphi(\Omega)^{\prime}$ be a projection. $\varphi$ is called c.n. $(W)$ (completely non- $(W))$ on $Q H$ if the only subprojection of $Q$ that $(W)$-reduces $\varphi$ is zero.

(2.1) Theorem (cf. [7, Proposition 1, Theorem 5]). If $(W)$ is hereditary and $\mathscr{P}$ is strongly closed and satisfies the conditions

(a) if $E, F \in \mathscr{P}, E F=0$, then $E+F \in \mathscr{P}$, and

(b) if $E \in \mathscr{P}$ and $F \in \varphi(\Omega)^{\prime}$ is a projection equivalent to $E$ with respect to $W^{*}(\varphi(\Omega))^{\prime}$, then $F \in \mathscr{P}$,

then $P=\sup \mathscr{P}$ exists, $P$ is the largest $(W)$-reducing projection for $\varphi$, and $P$ belongs to the center of $W^{*}(\varphi(\Omega))$. Moreover, $\varphi$ is c.n. $(W)$ on $(I-P) H$, and the decomposition $\varphi(u)=\varphi(u)_{\mid P H} \oplus \varphi(u)_{\mid(I-P) H}$ is unique.

The above decomposition is called the $(W)$-canonical decomposition of $\varphi$.

In what follows canonical decompositions will be constructed for an arbitrary number of functions and an arbitrary number of properties. The number of functions does not need to equal the number of properties. To make the exposition clear, attention will be restricted to two functions and two properties.

Let $\Omega_{j}$ be a set, let $\varphi_{j}: \Omega_{j} \rightarrow B(H)$ be a function, and let $\left(W_{j}\right)$ be a property concerning $\varphi_{j}, j=1,2$. One shall say that $\varphi_{1}, \varphi_{2}$ have the $\left(W_{1}, W_{2}\right)$ canonical decomposition if there exist projections $E_{1}, E_{2}, E_{3}, E_{4} \in B(H)$ such that

(C1) $E_{k} \in\left(\varphi_{1}\left(\Omega_{1}\right) \cup \varphi_{2}\left(\Omega_{2}\right)\right)^{\prime}, k=1, \ldots, 4$;

(C2) $E_{i} E_{k}=0$ if $i \neq k$;

(C3) $I=E_{1}+E_{2}+E_{3}+E_{4}$;

(C4) $E_{1}$ is a $\left(W_{j}\right)$-reducing projection for $\varphi_{j}, j=1,2$;

(C5) $E_{2}$ is a $\left(W_{1}\right)$-reducing projection for $\varphi_{1}$ and $\varphi_{2}$ is c.n. $\left(W_{2}\right)$ on $E_{2} H$; 
(C6) $\varphi_{1}$ is c.n. $\left(W_{1}\right)$ on $E_{3} H$ and $E_{3}$ is a $\left(W_{2}\right)$-reducing projection for $\varphi_{2}$;

(C7) $\varphi_{j}$ is c.n. $\left(W_{j}\right)$ on $E_{4} H, j=1,2$.

This notation will be preserved. The following theorem is the main result of this section.

(2.2) Theorem. Let $\Omega_{j}$ be a set, let $\varphi_{j}: \Omega_{j} \rightarrow B(H)$ be a function, and let $\left(W_{j}\right)$ be a hereditary property concerning $\varphi_{j}, j=1,2$. Suppose that there exists the largest $\left(W_{j}\right)$-reducing projection $P_{j}$ for $\varphi_{j}$ and that $P_{j}$ is in the center of $W^{*}\left(\varphi_{j}\left(\Omega_{j}\right)\right), j=1,2$. Then $\varphi_{1}, \varphi_{2}$ have the $\left(W_{1}, W_{2}\right)$-canonical decomposition if and only if $P_{j} \in\left(\varphi_{1}\left(\Omega_{1}\right) \cup \varphi_{2}\left(\Omega_{2}\right)\right)^{\prime}, j=1,2$. In such a case, $P_{1}=E_{1}+E_{2}, P_{2}=E_{1}+E_{3}$, and $E_{1}=P_{1} P_{2}, E_{2}=P_{1}\left(I-P_{2}\right)$, $E_{3}=\left(I-P_{1}\right) P_{2}, E_{4}=\left(I-P_{1}\right)\left(I-P_{2}\right)$. The decomposition is unique.

Proof. Uniqueness follows from the uniqueness of $P_{1}, P_{2}$ for $\varphi_{1}, \varphi_{2}$, respectively, by Theorem 2.1 and from the last four equalities. It should be mentioned that hereditariness is the main property responsible for uniqueness (cf. [7, (A)]). If $P_{1}, P_{2} \in\left(\varphi_{1}\left(\Omega_{1}\right) \cup \varphi_{2}\left(\Omega_{2}\right)\right)^{\prime}$, then $P_{1} P_{2}=P_{2} P_{1}$, because $P_{j}$ is in the center of $W^{*}\left(\varphi_{j}\left(\Omega_{j}\right)\right), j=1,2$. The last four equalities in the statement of the theorem now define projections that satisfy $(\mathrm{C} 1)-(\mathrm{C} 7)$.

Conversely, suppose that $\varphi_{1}, \varphi_{2}$ have the $\left(W_{1}, W_{2}\right)$-canonical decomposition. Note that $E_{k} P_{j}=P_{j} E_{k}, j=1,2, k=1, \ldots, 4$. For, $P_{j} \in W^{*}\left(\varphi_{j}\left(\Omega_{j}\right)\right)$ and $E_{k} \in W^{*}\left(\varphi_{j}\left(\Omega_{j}\right)\right)^{\prime}$ by $(\mathrm{C} 1)$. Since $E_{1}, E_{2}$ are $\left(W_{1}\right)$-reducing projections for $\varphi_{1}$ and $P_{1}$ is the largest one with this property, $E_{1}+E_{2} \leq P_{1}$. Now, $P_{1} E_{3} \leq P_{1}$ is a $\left(W_{1}\right)$-reducing projection for $\varphi_{1}$, because $\left(W_{1}\right)$ is hereditary. On the other hand, $P_{1} E_{3} \leq E_{3}$ and $\varphi_{1}$ is c.n. $\left(W_{1}\right)$ on $E_{3} H$. Hence $P_{1} E_{3}=0$. In a similar manner one proves that $P_{1} E_{4}=0$. Thus, by (C3), $P_{1} \leq E_{1}+E_{2}$. An analogous argument justifies that $P_{2}=E_{1}+E_{3}$. Hence $P_{j} \in\left(\varphi_{1}\left(\Omega_{1}\right) \cup \varphi_{2}\left(\Omega_{2}\right)\right)^{\prime}$ by $(\mathrm{C} 1)$.

(2.3) Corollary. Let $\Omega_{j}, \varphi_{j},\left(W_{j}\right)$, and $P_{j}$ be as in Theorem (2.2). Suppose $P_{j} \in W^{*}\left(\varphi_{j}\left(\Omega_{j}\right)\right), j=1,2$. If $\varphi_{1}\left(\Omega_{1}\right) \subset W^{*}\left(\varphi_{2}\left(\Omega_{2}\right)\right)^{\prime}$, then $\varphi_{1}, \varphi_{2}$ have the $\left(W_{1}, W_{2}\right)$-canonical decomposition.

Proof. It is clear that $W^{*}\left(\varphi_{1}\left(\Omega_{1}\right)\right) \subset W^{*}\left(\varphi_{2}\left(\Omega_{2}\right)\right)^{\prime}$. Also, by the Double Commutant Theorem $W^{*}\left(\varphi_{2}\left(\Omega_{2}\right)\right) \subset W^{*}\left(\varphi_{1}\left(\Omega_{1}\right)\right)^{\prime}$. Since $P_{j} \in W^{*}\left(\varphi_{j}\left(\Omega_{j}\right)\right)$, one concludes that $P_{j} \in\left(W^{*}\left(\varphi_{1}\left(\Omega_{1}\right)\right) \cup W^{*}\left(\varphi_{2}\left(\Omega_{2}\right)\right)\right)^{\prime}, j=1,2$. Use Theorem (2.2).

For one function $\varphi\left(=\varphi_{1}=\varphi_{2}\right)$ and several properties one immediately has (2.4) Corollary. Let $\varphi: \Omega \rightarrow B(H)$ be a function, and let $\left(W_{1}\right),\left(W_{2}\right)$ be hereditary properties concerning $\varphi$. If there exists the largest $\left(W_{j}\right)$-reducing projection $P_{j}$ for $\varphi$ and $P_{j}$ is in the center of $W^{*}(\varphi(\Omega)), j=1,2$, then $\varphi$ has the $\left(W_{1}, W_{2}\right)$-canonical decomposition.

Proof. Obvious by Theorem (2.2), because $P_{1}, P_{2} \in \varphi(\Omega)^{\prime}$.

In an even more particular case of $\varphi_{1}=\varphi_{2}$ and $\left(W_{1}\right)=\left(W_{2}\right)$, the $\left(W_{1}, W_{2}\right)$ canonical decomposition of $\varphi_{1}, \varphi_{2}$ reduces to the $\left(W_{1}\right)$-canonical decomposition of $\varphi_{1}$. It is known that the $(W)$-canonical decomposition of $\varphi$ sometimes does not exist (cf., e.g., [9] for $(W)=$ (symmetric commutant), [8] for $(W)=($ antisymmetric $))$. Thus the $\left(W_{1}, W_{2}\right)$-canonical decomposition of $\varphi_{1}, \varphi_{2}$ may not exist either. An example of two functions (operators) for which 
the $(W, W)$-canonical decomposition does not exist, where $(W)=$ (unitary), even though the individual $(W)$-canonical decomposition (in this case, plainly, the Wold decomposition of an isometry) exists for each function (operator), can be found in [6, Example 1]. In this example the assumption $P_{j} \in$ $\left(\varphi_{1}\left(\Omega_{1}\right) \cup \varphi_{2}\left(\Omega_{2}\right)\right)^{\prime}, j=1,2$, of Theorem (2.2) fails to be satisfied.

To explain how to use the above results for operators rather than operatorvalued functions, consider the following particular situation. Let $T_{1}, T_{2} \in$ $B(H)$. Let $\Omega$ be an arbitrary set, and let $\varphi_{j}: \Omega \rightarrow B(H)$ be the constant function defined by $\varphi_{j}(u)=T_{j}, u \in \Omega, j=1,2$. The operators $T_{1}, T_{2}$ will be said to have the $\left(W_{1}, W_{2}\right)$-canonical decomposition if the functions $\varphi_{1}, \varphi_{2}$ have the $\left(W_{1}, W_{2}\right)$-canonical decomposition. In this simpler case, often sufficient and more convenient for applications to operators (e.g., in the next section), Theorem (2.2) reads as follows:

(2.5) Corollary. Let $T_{j} \in B(H)$, and let $\left(W_{j}\right)$ be a property concerning $T_{j}$, $j=1,2$. Suppose there is the largest $\left(W_{j}\right)$-reducing projection $P_{j}$ for $T_{j}$ and $P_{j} \in W^{*}\left(T_{j}\right), j=1,2$. Then $T_{1}, T_{2}$ have the $\left(W_{1}, W_{2}\right)$-canonical decomposition if and only if $P_{j} \in\left\{T_{1}, T_{2}\right\}^{\prime}, j=1,2$.

Operators $T_{1}, T_{2} \in B(H)$ doubly commute if $T_{1} \in W^{*}\left(T_{2}\right)^{\prime}$ or, equivalently, $W^{*}\left(T_{1}\right) \subset W^{*}\left(T_{2}\right)^{\prime}$. Corollary (2.3) interpreted for operators concerns exactly this situation and reads

(2.6) Corollary. Let $T_{j},\left(W_{j}\right)$, and $P_{j}$ be as in Corollary (2.5). Suppose $P_{j} \in$ $W^{*}\left(T_{j}\right), j=1,2$. If $T_{1}, T_{2}$ doubly commute, then $T_{1}, T_{2}$ have the $\left(W_{1}, W_{2}\right)$ canonical decomposition.

The existence of the $\left(W_{1}, W_{2}\right)$-canonical decomposition does not imply, however, that the operators doubly commute. Here is an example.

(2.7) Example. Take a nonunitary isometry $V \in B(H), T_{1}=T_{2}=V$, and $\left(W_{1}\right)=\left(W_{2}\right)=$ (unitary). Then the $\left(W_{1}, W_{2}\right)$-canonical decomposition of $T_{1}, T_{2}$ exists, and it is simply the Wold decomposition of $V$. All assumptions of Corollary (2.5) are satisfied because the largest (unitarily)-reducing projection $P$ for $V$ belongs to $W^{*}(V)$ by Theorem (2.1) and, clearly, $P_{1}=P_{2}=$ $P \in\left\{T_{1}, T_{2}\right\}^{\prime}$. Since $V$ is not unitary, $T_{1}, T_{2}$ do not doubly commute.

The interpretation of Corollary (2.4) for a single operator and several properties is left to the reader.

In general, to check whether there is the largest $(W)$-reducing projection $P$ for $T \in B(H)$ and $P \in W^{*}(T)$, one uses Theorem (2.1). For example, if $p$ is a polynomial in two noncommuting variables and the property $(W)$ is defined by the condition $p\left(S, S^{*}\right)=0$ (or $p\left(S, S^{*}\right) \geq 0$ ) for Hilbert space operators $S$, then the assumptions of Theorem (2.1) are satisfied, which can be deduced from results of [7] (cf. [4]). In particular, let

$$
p\left(S, S^{*}\right)=S^{*} S-S S^{*} .
$$

Then Corollary (2.6) implies the following result needed in the next section:

(2.8) Corollary. If $T_{1}, T_{2} \in B(H)$ doubly commute, then they have the (normal, normal)-canonical decomposition. 


\section{A MODEL OF A FAMILY}

\section{OF DOUBLY COMMUTING QUASINORMAL OPERATORS}

In this section we are going to construct a model of two doubly commuting quasinormal operators. For the sake of clarity we restrict our attention to two operators only. Our model, however, can be written up for any number of doubly commuting quasinormal operators.

Recall that an operator $T \in B(H)$ is quasinormal if $T$ commutes with $T^{*} T$. In the literature, for example [5,10], jointly quasinormal families of operators are also considered. A family $Q$ of operators is called jointly quasinormal if $C$ commutes with $D$ and $D^{*} D$ for all $C$ and $D$ in $Q$. A family of quasinormal operators that doubly commute is a jointly quasinormal family (cf. Lemma (3.3)). The opposite, however, fails, which can be seen considering two commuting isometries that do not doubly commute.

Unfortunately, we are not able to present a model for every quasinormal family, because our model relies on the canonical decomposition of families of operators developed in $\S 2$. Namely, jointly quasinormal families need not have (normal, normal, ... )-canonical decomposition. To see this, consider Stocinski's Example 1 in [6]. The two isometries in this example commute; thus, they are a jointly quasinormal family, but they do not have the (normal, normal)-canonical decomposition, (cf. remarks after Corollary (2.4)).

Let us consider quasinormal operators $T_{1}, T_{2} \in B(H)$ and assume that they doubly commute. According to Corollary (2.8) they have the (normal, normal)canonical decomposition. Therefore, in order to construct a model of these operators it is enough to consider two cases:

Case 1. $T_{1}$ is a normal operator, and $T_{2}$ is a c.n. normal operator.

Case 2. Both $T_{1}$ and $T_{2}$ are c.n. normal operators. Recall that an isometry $V \in B(H)$ is a shift (a c.n. unitary isometry) if $V^{m} M \perp V^{n} M$ for $m \neq$ $n, m, n \in \mathbb{N}$, and $H=\bigoplus_{n \in \mathbb{N}} V^{n} M$, where $M=(V H)^{\perp}$. Throughout the remainder of this paper $l_{+}^{2}$ is the Hilbert space of all square summable complex sequences with the canonical base $\left\{e_{0}, e_{1}, e_{2}, \ldots\right\}$, and $U_{+}$is the unilateral shift of multiplicity one in $l_{+}^{2}$.

Case 1.

(3.1) Proposition. Let $T_{1} \in B(H)$ be a c.n. normal, quasinormal operator, and let $T_{2} \in B(H)$ be a normal operator commuting with $T_{1}$. Then there exist a Hilbert space $M$, a positive operator $Q \in B(M)$ with $\operatorname{ker} Q=\{0\}$, and a normal operator $N \in B(M)$ commuting with $Q$ such that the pair $T_{1}$, $T_{2} \in B(H)$ is unitarily equivalent to the pair $U_{+} \otimes Q, I \otimes N \in B\left(l_{+}^{2} \otimes M\right)$.

Proof. Let $T_{1}=V P$ be the polar decomposition of $T_{1}$. Since $T_{1}$ is quasinormal and c.n. normal, $\operatorname{ker} T_{1}=\{0\}$. Thus $\operatorname{ker} P=\{0\}$ and $V$ is an isometry. Moreover, $V$ is a shift (cf. [2] or [3, Chapter II, proof of Theorem 3.2]). Let $M=(V H)^{\perp}$. Since $V$ is a shift, the mapping $\phi: l_{+}^{2} \otimes M \rightarrow H$ defined by $\phi\left(\sum_{n \in \mathbb{N}} e_{n} \otimes x_{n}\right)=\sum_{n \in \mathbb{N}} V^{n} x_{n}, x_{n} \in M, n \in \mathbb{N}$, is unitary. It is known that $\phi\left(U_{+} \otimes I\right)=V \phi$. Since $T_{1}$ is quasinormal, $P$ commutes with $V$, and thus $M$ reduces $P$. Let $Q=P_{\mid M}$. One checks easily that $\phi(I \otimes Q)=P \phi$. Until now we have merely given a short proof of the main result of [2, Theorem 1]. For a proof that does not use the tensor product language the reader may consult [ 3 , Chapter II, Theorem 3.2]. 
Now let us turn to $T_{2}$ which is a normal operator commuting with $T_{1}$. By Fuglede's theorem $T_{2} \in W^{*}\left(T_{1}\right)^{\prime}$. It is known that

(3.2) Both factors in the polar decomposition of an operator belong to the von Neumann algebra generated by that operator,

which, for the partial isometry factor, is a consequence of the Double Commutant Theorem (cf., e.g., [1, Corollary, p. 7]). Hence $T_{2}$ commutes with $V$ and $V^{*}$, and thus $M$ reduces $T_{2}$. Let $N=T_{2 \mid M}$. Since $T_{2}$ commutes also with $P, N$ commutes with $Q$. Also, $\operatorname{ker} Q=\{0\}$, because $\operatorname{ker} P=\{0\}$. Finally, if $x_{n} \in M, n \in \mathbb{N}$, then

$$
\begin{aligned}
\phi(I \otimes N)\left(\sum e_{n} \otimes x_{n}\right) & =\phi\left(\sum e_{n} \otimes N x_{n}\right)=\sum V^{n} N x_{n}=T_{2} \sum V^{n} x_{n} \\
& =T_{2} \phi\left(\sum e_{n} \otimes x_{n}\right),
\end{aligned}
$$

which proves that $\phi(I \otimes N)=T_{2} \phi$.

The next lemma, which will be needed in Case 2, is of interest on its own, because it clarifies the distinction between doubly commuting quasinormal operators and a jointly quasinormal family.

(3.3) Lemma. Let $T_{j}=V_{j} P_{j}$ be a polar decomposition, unique under the assumption $\operatorname{ker} V_{j}=\operatorname{ker} P_{j}$, of an operator $T_{j} \in B(H), j=1,2$. The following conditions are equivalent.

(a) $T_{1}, T_{2}$ are quasinormal, and they doubly commute;

(b) $T_{1}, T_{2}$ are jointly quasinormal, and $V_{1} V_{2}^{*}=V_{2}^{*} V_{1}$;

(c) $V_{1}, V_{2}, P_{1}, P_{2}$ commute each other, and $V_{1} V_{2}^{*}=V_{2}^{*} V_{1}$.

Proof. (b) $\Leftrightarrow$ (c) in Lemma 3.9(b) of [10] (cf. also [5, proof of Theorem 4.5]. (c) $\Rightarrow(\mathrm{a})$ is clear. Now we prove $(\mathrm{a}) \Rightarrow$ (c). $V_{j} P_{j}=P_{j} V_{j}$, because $T_{j}$ is quasinormal, $j=1,2$. Since $T_{1}, T_{2}$ doubly commute, $W^{*}\left(T_{1}\right) \subset W^{*}\left(T_{2}\right)^{\prime}$. By (3.2) we conclude that $V_{1}, V_{2}, P_{1}, P_{2}$ commute each other.

Moreover, $V_{1} V_{2}^{*} P_{1} P_{2}=V_{1} P_{1} P_{2} V_{2}^{*}=T_{1} T_{2}^{*}=T_{2}^{*} T_{1}=P_{2} V_{2}^{*} V_{1} P_{1}=V_{2}^{*} V_{1} P_{1} P_{2}$, which proves that $V_{1} V_{2}^{*} x=V_{2}^{*} V_{1} x$ if $x \in\left(P_{1} P_{2} H\right)^{-}=$the closure of $P_{1} P_{2} H=$ $\left(\operatorname{ker} P_{1} P_{2}\right)^{\perp}$.

Now we shall prove that $V_{1} V_{2}^{*} x=V_{2}^{*} V_{1} x=0$ for $x \in \operatorname{ker} P_{1} P_{2}$. Since $T_{j}$ is quasinormal, $\operatorname{ker} T_{j}=\operatorname{ker} P_{j}$ reduces $T_{j}, j=1,2$. Since $T_{1}, T_{2}$ doubly commute, $\operatorname{ker} P_{j}$ reduces $T_{i}, i, j=1,2$. By (3.2) again, $\operatorname{ker} P_{j}$ reduces $V_{i}, i, j=1,2$.

Let $x \in \operatorname{ker} P_{1}=\operatorname{ker} V_{1}$. Then $V_{1} x=0$ and $V_{2}^{*} x \in \operatorname{ker} P_{1}=\operatorname{ker} V_{1}$. Thus $V_{1} V_{2}^{*} x=V_{2}^{*} V_{1} x=0$, i.e., $V_{1} V_{2}^{*}=V_{2}^{*} V_{1}=0$ on $\operatorname{ker} P_{1}$.

One proves similarly that $V_{1}^{*} V_{2}=V_{2} V_{1}^{*}=0$ on $\operatorname{ker} P_{2}$, which is equivalent to $V_{2}^{*} V_{1}=V_{1} V_{2}^{*}=0$ on $\operatorname{ker} P_{2}$, because $\operatorname{ker} P_{2}$ reduces $V_{1}, V_{2}$. Since $P_{1}$, $P_{2}$ are commuting positive operators, it follows from the spectral theorem that ker $P_{1} P_{2}=\left(\operatorname{ker} P_{1}+\operatorname{ker} P_{2}\right)^{-}$, which completes the proof.

Case 2. We begin our discussion of Case 2 with the following

(3.4) Proposition. Let $V_{1}, V_{2} \in B(H)$ be shifts that doubly commute. Let $M=\left(V_{1} H\right)^{\perp} \cap\left(V_{2} H\right)^{\perp}$. Then the pair $V_{1}, V_{2} \in B(H)$ is unitarily equivalent to the pair $U_{+} \otimes I \otimes I, I \otimes U_{+} \otimes I \in B\left(l_{+}^{2} \otimes l_{+}^{2} \otimes M\right)$. 
Proof. Recall that the set $\left\{e_{m} \otimes e_{n}: m, n \in \mathbb{N}\right\}$ is an orthonormal base of $l_{+}^{2} \otimes l_{+}^{2}$. It follows from Theorem 1 of [6] that $V_{1}^{k} V_{2}^{l} M \perp V_{1}^{m} V_{2}^{n} M$ for $(k, l) \neq$ $(m, n), k, l, m, n \in \mathbb{N}$, and that $H=\bigoplus_{m, n \in \mathbb{N}} V_{1}^{m} V_{2}^{n} M$. Hence the mapping $\psi: l_{+}^{2} \otimes l_{+}^{2} \otimes M \rightarrow H$ defined by

$$
\psi\left(\sum_{m, n \in \mathbb{N}} e_{m} \otimes e_{n} \otimes x_{m n}\right)=\sum_{m, n \in \mathbb{N}} V_{1}^{m} V_{2}^{n} x_{m n}, \quad x_{m n} \in M,
$$

is unitary. Take $x_{m n} \in M, m, n \in \mathbb{N}$, and define $y_{m n}=x_{m-1, n}$ if $m>0$, $y_{0 n}=0, n \in \mathbb{N}$. Then

$$
\begin{aligned}
\psi\left(U_{+}\right. & \otimes I \otimes I)\left(\sum e_{m} \otimes e_{n} \otimes x_{m n}\right)=\psi\left(\sum e_{m+1} \otimes e_{n} \otimes x_{m n}\right) \\
& =\psi\left(\sum e_{m+1} \otimes e_{n} \otimes y_{m+1, n}\right)=\psi\left(\sum e_{m} \otimes e_{n} \otimes y_{m n}\right) \\
& =\sum V_{1}^{m} V_{2}^{n} y_{m n}=\sum V_{1}^{m+1} V_{2}^{n} y_{m+1, n}=\sum V_{1}^{m+1} V_{2}^{n} x_{m n} \\
& =V_{1} \sum V_{1}^{m} V_{2}^{n} x_{m n}=V_{1} \psi\left(\sum e_{m} \otimes e_{n} \otimes x_{m n}\right),
\end{aligned}
$$

which proves that $\psi\left(U_{+} \otimes I \otimes I\right)=V_{1} \psi$. In a similar manner one proves that $\psi\left(I \otimes U_{+} \otimes I\right)=V_{2} \psi$, "shifting" the second index in $x_{m n}$ and, at the end, using again the assumption that $V_{1}, V_{2}$ (doubly) commute.

(3.5) Proposition. Let $T_{1}, T_{2} \in B(H)$ be c.n. normal, quasinormal operators that doubly commute. Then there exist a Hilbert space $M$ and positive, commuting operators $Q_{1}, Q_{2} \in B(M)$ with $\operatorname{ker} Q_{j}=\{0\}, j=1,2$, such that the pair $T_{1}, T_{2} \in B(H)$ is unitarily equivalent to the pair $U_{+} \otimes I \otimes Q_{1}$, $I \otimes U_{+} \otimes Q_{2} \in B\left(l_{+}^{2} \otimes l_{+}^{2} \otimes M\right)$.

Proof. Let $T_{j}=V_{j} P_{j}$ be the polar decomposition of $T_{j}$. Then $\operatorname{ker} P_{j}=\{0\}$ and $V_{j}$ is a shift, $j=1,2$ (cf. the beginning of the proof of Proposition (3.1)). Since $T_{1}, T_{2}$ doubly commute, the operators $P_{1}, P_{2}, V_{1}, V_{2}$ commute each other (cf. Lemma (3.3)). Hence $M=\left(V_{1} H\right)^{\perp} \cap\left(V_{2} H\right)^{\perp}$ reduces $P_{j}$. Let $Q_{j}=P_{j_{\mid M}}$. Clearly, $\operatorname{ker} Q_{j}=\{0\}, j=1,2$, and $Q_{1}$ and $Q_{2}$ commute. Now apply Proposition (3.4) to $V_{1}, V_{2}$, which doubly commute, by Lemma (3.3). Let $\psi: l_{+}^{2} \otimes l_{+}^{2} \otimes M \rightarrow H$ be the unitary mapping defined in the proof of Proposition (3.4). Take $x_{m n} \in M, m, n \in \mathbb{N}$. Then

$$
\begin{aligned}
\psi(I & \left.\otimes I \otimes Q_{j}\right)\left(\sum e_{m} \otimes e_{n} \otimes x_{m n}\right)=\psi\left(\sum e_{m} \otimes e_{n} \otimes Q_{j} x_{m n}\right) \\
& =\sum V_{1}^{m} V_{2}^{n} Q_{j} x_{m n}=P_{j} \sum V_{1}^{m} V_{2}^{n} x_{m n}=P_{j} \psi\left(\sum e_{m} \otimes e_{n} \otimes x_{m n}\right),
\end{aligned}
$$

which proves that $\psi\left(I \otimes I \otimes Q_{j}\right)=P_{j} \psi, j=1,2$.

This, together with Proposition (3.4), finishes the proof.

The final result summarizes all the above results and gives a complete model of two doubly commuting quasinormal operators.

(3.6) Theorem. Let $T_{1}$ and $T_{2}$ be doubly commuting quasinormal operators. Then the pair $T_{1}, T_{2}$ is unitarily equivalent to the pair

$$
\begin{aligned}
& M_{1} \oplus\left(I \otimes N_{1}\right) \oplus\left(U_{+} \otimes P_{1}\right) \oplus\left(U_{+} \otimes I \otimes Q_{1}\right), \\
& M_{2} \oplus\left(U_{+} \otimes P_{2}\right) \oplus\left(I \otimes N_{2}\right) \oplus\left(I \otimes U_{+} \otimes Q_{2}\right),
\end{aligned}
$$


where

(a) $M_{1}, M_{2}, N_{1}$, and $N_{2}$ are normal operators,

(b) $P_{1}, P_{2}, Q_{1}$, and $Q_{2}$ are positive operators with zero kernels,

(c) $M_{1}$ and $M_{2} ; P_{1}$ and $N_{2} ; N_{1}$ and $P_{2}$; and $Q_{1}$ and $Q_{2}$ commute.

\section{REFERENCES}

1. W. B. Arveson, An invitation to $C^{*}$-algebras, Springer-Verlag, New York, Heidelberg, and Berlin, 1976.

2. A. Brown, On a class of operators, Proc. Amer. Math. Soc. 4 (1953), 723-728.

3. J. B. Conway, The theory of subnormal operators, Math. Surveys Monographs, vol. 36, Amer. Math. Soc., Providence, RI, 1991.

4. M. Fujii, M. Kajiwara, Y. Kato, and F. Kubo, Decompositions of operators in Hilbert spaces, Math. Japon. 21 (1976), 117-120.

5. A. Lubin, Weighted shifts in commuting normal extensions, J. Austral. Math. Soc. Ser. A 27 (1979), 17-26.

6. M. Slocinski, On the Wold-type decomposition of a pair of commuting isometries, Ann. Polon. Math. 37 (1980), 255-262.

7. W. Szymański, Decompositions of operator-valued functions in Hilbert spaces, Studia Math. 50 (1974), 265-280.

8. __ Antisymmetric operator algebras. II, Ann. Polon. Math. 37 (1980), 299-311.

9. _ On the "symmetric commutant"-canonical decompositions of families of Hilbert space operators, Ark. Mat. 21 (1983), 205-215.

10. _ Dilations and subnormality, Proc. Amer. Math. Soc. 101 (1987), 251-259.

11. H. Wold, $A$ study in analysis of stationary time series, Almqvist and Wiksells, Uppsala, 1983; 2nd ed., 1954 . 17551

Department of Mathematics, Millersville University, Millersville, Pennsylvania

E-mail address: xcatepil@mu2.millersv.edu

Institute of Mathematics, University of Agriculture, Ul.18 StyczNia 6, 30-045 Kraków, Poland

Department of Mathematics and Computer Science, West Chester University, West Chester, Pennsylvania 19383

E-mail address: wszymans@wcu.bitnet 